\title{
Analysis of Slot Characteristics in Slotted Single-Mode Semiconductor Lasers Using the 2-D Scattering Matrix Method
}

\author{
Q. Y. Lu, W. H. Guo, R. Phelan, D. Byrne, J. F. Donegan, P. Lambkin, and B. Corbett
}

\begin{abstract}
We use the two-dimensional (2-D) scattering matrix method (SMM) to analyze the slot characteristics in slotted singlemode semiconductor lasers and compare the results with those calculated by the one-dimensional transfer matrix method (TMM). The analysis shows that the 2-D SMM is required to accurately account for the measured results. Using the 2-D SMM simulation, we find that there is almost no reflection at the interface from slot to waveguide while a large reflection exists at the interface from waveguide to slot, and the power loss is much larger than the power reflected. For a single slot, the slot width has little influence on the slot reflectivity, which coincides with the measured results. The reflection and transmission of the slot are found to be exponentially dependent on the slot depth.
\end{abstract}

Index Terms-Perfectly matched absorption layers (PMLs), scattering matrix method, semiconductor lasers, single-mode laser, transfer matrix method.

\section{INTRODUCTION}

D ISTRIBUTED feedback (DFB) lasers as archetypal single-mode semiconductor lasers have achieved great success. However, fabricating these lasers requires both highresolution processing and complex regrowth steps. On the other hand, single-mode lasers can be realized by distributing reflective defects (in the following we call them slots) into conventional Fabry-Perot (FP) laser cavities [1]-[3]. By carefully optimizing the slot positions and slot number, lasing with a side-mode suppression ratio (SMSR) of more than $40 \mathrm{~dB}$ has been achieved [2]. For optimization of such lasers, first we need to make clear the characteristics of a single slot, most importantly the reflection and scattering loss caused by the slot. Experimental work has been carried out in which the slot reflectivity was measured [4] and the slot loss was estimated [3]. Theoretical work on the effect of slots was carried out in the 1980s by Coldren and co-workers. In [5], the slots (grooves in those papers) are separated into two different slot-depth cases dealing with two different models and more emphasis is laid on the deep slot case. For the shallow slot, the model

Manuscript received August 21, 2006; revised October 16, 2006. This work was supported by SFI under its CSET Centre for Telecommunication Value Driven Research (CTVR), under Grant 03/IE3/I405.

Q. Y. Lu, W. H. Guo, R. Phelan, D. Byrne, and J. F. Donegan are with the Semiconductor Photonics Group, School of Physics and Centre for Telecommunication Value-Driven Research (CTVR), Trinity College, Dublin 2, Ireland (e-mail: luqi@tcd.ie).

P. Lambkin and B. Corbett are with the Photonics Group and Centre for Telecommunication Value-Chain Driven Research (CTVR), Tyndall National Institute, Lee Maltings, Cork, Ireland.

Digital Object Identifier 10.1109/LPT.2006.887328

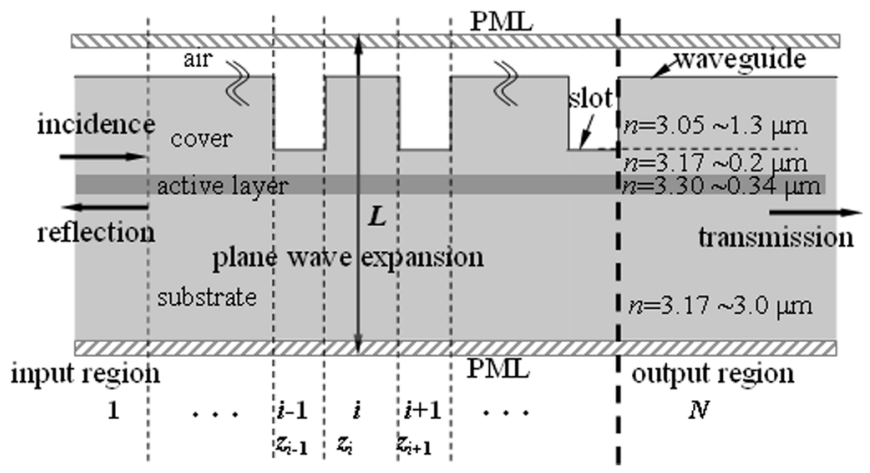

Fig. 1. Schematic diagram of a 2-D multiple-slot waveguide structure.

is intuitive which assumes that the reflected and transmitted modes are excited by the reflected tail and the remainder of the incident wave, respectively. Now as single-mode lasers with shallow slots have achieved excellent results, the analysis of slots is now again an area of great interest especially with the use of stricter numerical analysis techniques. In [2], the one-dimensional (1-D) transfer matrix method (TMM) was used to analyze lasers with multiple slots. The analysis is straightforward but unfortunately the scattering loss can not be determined. Furthermore, the reflection coefficient from the TMM model does not reproduce the experimental results [4]. In this letter, we use the two-dimensional (2-D) scattering matrix method (SMM) [6] to solve the slotted laser problem. It has the benefit of the TMM method as it can easily tackle the whole laser structure with multiple slots, and moreover, because it analyzes the transverse direction and the propagation direction simultaneously it can predict the loss caused by the slot. We use the 2-D SMM model mainly to analyse the characteristics of a single shallow slot but also it can be easily extended to analyze deep slots and multiple-slot structures.

\section{THEORY}

For generality we start with a multiple-slot structure. As shown in Fig. 1, a 2-D multiple-slot waveguide can be separated into $N$ uniform sections including the input and output regions. Hereafter, the subscript $i, i=1,2, \ldots, N$, is used to refer to the $i$ th section. The 2-D SMM model is used to compute the field reflected and coupled into the input waveguide and also the field transmitted and coupled into the output waveguide, which are defined as the reflection and the transmission of the group 
of slots. In the model, the actual waveguide is extended periodically with period $L$ in the transverse direction as shown in Fig. 1. At the boundary of the new periodic structure, perfectly matched absorption layers (PMLs) [7] are employed to absorb the outgoing waves. Considering the TE polarization case, the mode equation in the waveguide with the PML absorber can be expressed as

$$
\frac{1}{s_{x}} \frac{\partial}{\partial x} \frac{1}{s_{x}} \frac{\partial}{\partial x} E_{y}(x, z)+n^{2}(x, z) k_{0}^{2} E_{y}(x, z)=\beta^{2} E_{y}(x, z)
$$

where $k_{0}=2 \pi / \lambda$ is the wavenumber in vacuum, $s_{x}=1+$ $\sigma_{x} / j \omega \varepsilon_{0}, \sigma_{x}$ denoting the electronic conductivity results in the absorption and exists only within the PML region, $E_{y}$ is the $y$ component of the electric field which is uniform in the $y$ direction. Considering the periodic boundary condition we introduce complete, orthogonal, and normalized plane wave basis functions $\mid p>=\exp (-i 2 p \pi x / L) L^{-1 / 2}$, where $p$ is an integer and in practical calculations, $p$ is always truncated from $-M$ to $M, M$ corresponds to the highest spatial frequency used in the expansion. Using the basis functions, (1) can be transformed into a matrix form

$$
\begin{array}{r}
\sum_{q=-M}^{M}\left(\left\langle p\left|\frac{1}{s_{x}} \frac{\partial}{\partial x} \frac{1}{s_{x}} \frac{\partial}{\partial x}\right| q\right\rangle+k_{0}^{2}\left\langle p\left|n^{2}(x, z)\right| q\right\rangle\right) \\
\times\left\langle q \mid E_{y}(x, z)\right\rangle=\beta^{2}\left\langle p \mid E_{y}(x, z)\right\rangle
\end{array}
$$

where $\langle\mid\rangle$ represents the inner product. In the following, $\tilde{E}_{y}$ is used to substitute the vector $\left\langle q \mid E_{y}\right\rangle$ for simplicity. Since the refractive index distribution is piecewise uniform in the $z$ direction as shown in Fig. 1, the electric field in the $i$ th section can be expressed as the summation of forward and backward propagating waves

$$
\begin{aligned}
\tilde{E}_{y, i}(z)=\sum_{p=-M}^{M} \tilde{e}_{p, i}\left[\exp \left(-j \beta_{p, i}\left(z-z_{i}\right)\right) c_{p, i}^{+}\right. & \\
& \left.+\exp \left(j \beta_{p, i}\left(z-z_{i}\right)\right) c_{p, i}^{-}\right]
\end{aligned}
$$

where $\tilde{e}_{p, i}$ and $\beta_{p, j}^{2}$ are eigenvectors and eigenvalues of (2) in the $i$ th section, $c_{p, i}^{+}$and $c_{p, i}^{-}$are the forward and backward amplitudes, respectively. All uniform sections of the waveguide can be treated similarly. At the interface between two adjacent sections, the mode matching condition is implemented, which requires $E_{y}$ and $\mathrm{d} E_{y} / \mathrm{d} z$ to be continuous at the interface. These continuity conditions have the field amplitudes in adjacent sections connected. Through an iterative process, the connection between the field amplitudes in the input and output regions can be finally established through the scattering matrix technique

$$
\left[\begin{array}{c}
c_{p, 1}^{-} \\
c_{p, N}^{+}
\end{array}\right]=\left[\begin{array}{ll}
S_{11} & S_{12} \\
S_{21} & S_{22}
\end{array}\right]\left[\begin{array}{c}
c_{p, 1}^{+} \\
c_{p, N}^{-}
\end{array}\right] \equiv S\left[\begin{array}{c}
c_{p, 1}^{+} \\
c_{p, N}^{-}
\end{array}\right]
$$

where each $\mathrm{S}_{i, j}$ is a $2 M+1$ by $2 M+1$ matrix. The submatrix $\mathrm{S}_{11}$ and $\mathrm{S}_{21}$ are the reflection matrix and transmission matrix, respectively. The guided mode is given a subscript $g$ in the vector $c_{p, 1}^{+}$and $h$ in $c_{p, N}^{+}$. If we assume that the incident wave is
TABLE I

REFLECTION AND TRANSMISSION COEFFICIENTS AT SINGLE SLOT INTERFACES CAlCulated By 2-D SMM COMPARED WiTh 1-D TMM

\begin{tabular}{ccccccc}
\hline \multirow{2}{*}{ Interface } & \multicolumn{2}{c}{$|\boldsymbol{r}|$} & \multicolumn{2}{c}{$|\boldsymbol{t}|$} & \multicolumn{2}{c}{ Power loss (dB) } \\
\cline { 2 - 7 } & TMM & SMM & TMM & SMM & TMM & SMM \\
\hline WG to Slot & 0.004 & 0.0143 & 0.99 & 0.972 & 0 & 0.247 \\
\hline Slot to WG & 0.004 & 0.00037 & 0.99 & 0.972 & 0 & 0.247 \\
\hline
\end{tabular}

WG represents waveguide in table.

completely composed of the guided mode, we can obtain the reflection $r$ and transmission $t$ into the guided mode respectively as

$$
r=S_{11}(g, g) \quad, t=S_{21}(h, g) \text {. }
$$

\section{Single Slot Simulation}

In the following, we analyze the characteristics of a single slot. The device structure simulated is the same as that used in [3]. The slot is etched into the ridge with the depth equal to the ridge height. The slot width is taken as $1.0 \mu \mathrm{m}$ at first. Using the weighted-index method the three-dimensional structure is simplified into a 2-D structure as shown in Fig. 1 with all layer thickness and refractive index given. The reflection and transmission coefficients at the slot interfaces obtained from the 2-D SMM model are given in Table I together with the results from the 1-D TMM model. It is found that from the 2-D SMM model, there is little reflection at the interface from the slot region to the waveguide region while a relatively large reflection exists at the interface from the waveguide to the slot region. It is also shown that the power loss is much larger than the power reflection because the reflected power is $|r|^{2}$ but the power loss is $1-|r|^{2}-|t|^{2} \sim 2(1-|t|)$. The 1-D TMM model gives a much smaller reflection and predicts that the same reflection amplitude exists at both interfaces. Also, there is no loss predicted by the 1-D TMM model because just the guided mode is considered in this case. As shown in the above theoretical analysis, the plane wave basis was used for the expansion in the 2-D SMM model. Similarly, we could have chosen to use a local waveguide eigenmode expansion (eigenmode expansion method, EEM) [8]. Using an EEM-based commercial waveguide mode solver (FIMMWAVE), the calculated coefficients at single slot interfaces are 0.0141, 0.974 and 0.00034, 0.974, respectively, which agree very well with those obtained by the plane wave expansion as listed in Table I.

A simple model can explain the different coefficients at these two interfaces. Assume the power reflectivity caused by the index discontinuity is $R_{0} \equiv r_{0}^{2}$. As only part of the mode experiences this reflection (slot just partially etched into the waveguide), the reflected power is $R_{0} \Gamma, \Gamma$ is the optical confinement factor of the virtual layers that has been interrupted at the slot interfaces. Considering the reflected power needs to couple into the whole mode again, the power reflectivity for the whole mode now becomes $\Gamma^{2} R$, which results in the amplitude reflection $\Gamma r_{0}$. At the interfaces from waveguide to slot and from slot 


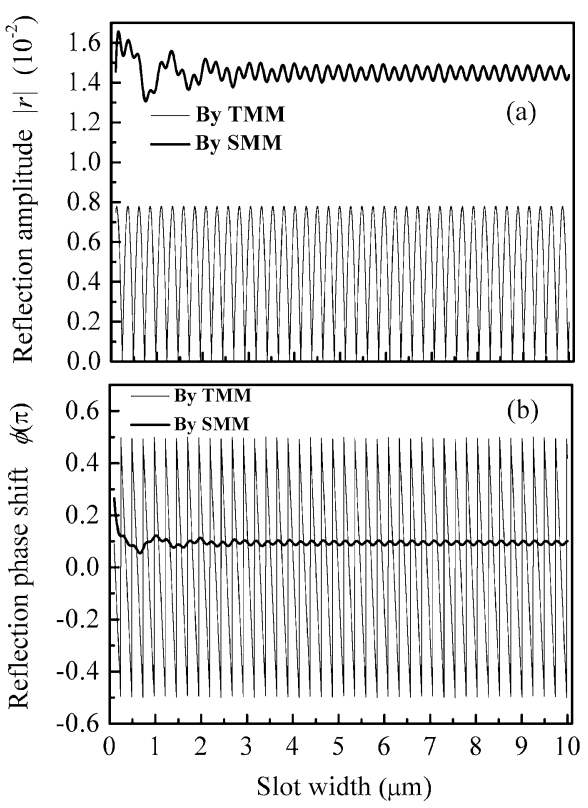

Fig. 2. (a) Reflection amplitude. (b) Reflection phase shift of a single slot versus slot width calculated by the 2-D SMM model and the 1-D TMM model.

to waveguide, $\Gamma$ is calculated to be 0.036 and 0.00075 , respectively. The latter is much smaller because at the slot-to-waveguide interface, the interrupted virtual layer is composed of air and little power is confined in it. $r_{0}$ has the same amplitude as 0.51 at both interfaces. The amplitude reflectivity calculated at both interfaces are 0.018 and 0.0004 , respectively, in good agreement with the 2-D SMM results. The above simplified model is essentially that used by Coldren and colleagues in [5].

We then calculated the reflection from the whole slot using the 2-D SMM model. The reflection and transmission amplitudes calculated are 0.014 and 0.96 , respectively. Accordingly, the loss introduced by a single slot for a cavity length $530 \mu \mathrm{m}$ is $\sim 1.4 \mathrm{~cm}^{-1}$. The reflectivity agrees well with the measured result in [4] but the loss is a little smaller than the value estimated in [3]. Fig. 2(a)-(b) shows the reflection amplitude and phase shift versus the slot width calculated using the 2-D SMM model as well as using the 1-D TMM model. It is seen that 1-D TMM model yields rapidly and periodically oscillating reflection amplitude and phase shift which evidently comes from the Fabry-Perot resonances. However, the 2-D SMM model predicts very different behavior: the reflection amplitude and phase shift show weak oscillation at small slot width and then almost settle down to a stable value. This is consistent with the observation in [4] and is due to the fact that almost no reflection is produced at the interface from the slot to the waveguide regions. In Fig. 3, we show the reflection and transmission variation with the slot depth. The results using Coldren's model [5] are given as well. We can see that for very shallow slots the results from the two models agree with each other very well. As the slot depth increases, Coldren's model still gives almost the same transmission as the 2-D SMM model, however, the reflection predicted is a little higher. In Coldren's model, the field tail at the waveguide-to-slot interface is assumed to be re-

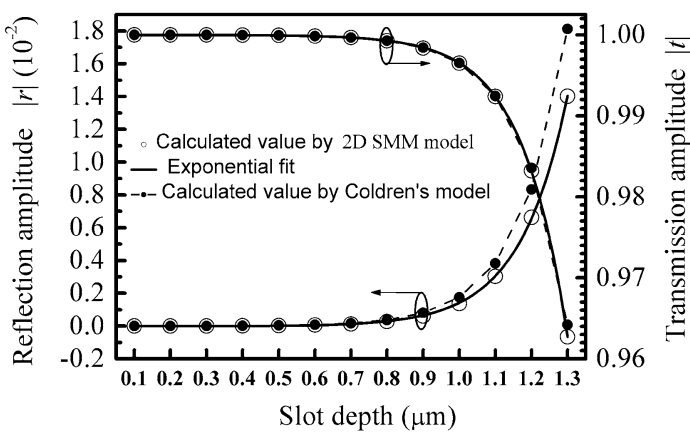

Fig. 3. Reflection and transmission amplitude of a single slot versus slot depth calculated by the 2-D SMM model and Coldren's model.

flected like a plane wave and the tail shape is assumed to be well preserved, which would overestimate the practical reflection. It is seen that the reflection increases while the transmission decreases exponentially as the slot depth increasing from 0.1 to $1.3 \mu \mathrm{m}$. The results of our 2-D SMM show that the slot depth should be precisely controlled in practice so as to obtain uniform reflections from different slots and also to improve the fabrication reproducibility.

\section{CONCLUSION}

We have analyzed the characteristics of a single slot in a slotted single-mode laser using the 2-D SMM model and compared the results yielded by the more traditional 1-D TMM method. From the 2-D SMM model, it is found that there is almost no reflection at the interface from slot to waveguide while a relatively large reflection exists at the interface from waveguide to slot. This asymmetry cancels the rapid reflection oscillation with slot width found in the TMM model. Further, the power loss is much larger than the power reflected. These results are consistent with those observed in experiments. It is also found that both the reflection and transmission exponentially depends on the slot depth.

\section{REFERENCES}

[1] B. Corbett and D. McDonald, "Single longitudinal mode ridge waveguides $1.3 \mu \mathrm{m}$ Fabry-Perot laser by modal perturbation," Electron. Lett., vol. 31, pp. 2181-2182, 1995.

[2] S. O'Brien and E. P. O'Reilly, "Theory of improved spectral purity in index patterned Fabry-Perot lasers," Appl. Phys. Lett., vol. 86, pp. 201101-1-201101-2, 2005.

[3] B. Corbett, C. Percival, and P. Lambkin, "Multiwavelength array of single-frequency stabilized Fabry-Perot lasers," IEEE J. Quantum Electron., vol. 41, no. 4, pp. 490-494, Apr. 2005.

[4] P. Lambkin, C. Percival, and B. Corbett, "Reflectivity measurements of intracavity defects in laser diodes," IEEE J. Quantum Electron., vol. 40, no. 1, pp. 10-17, Jan. 2004.

[5] L. A. Coldren, K. J. Ebeling, B. I. Miller, and J. A. Rentschler, "Single longitudinal mode operation of two-section GaInAsP/InP lasers under pulsed excitation," IEEE J. Quantum Electron., vol. QE-19, no. 6, pp. 1057-1062, Jun. 1983.

[6] E. Silberstein, P. Lalanne, J.-P. Hugonin, and Q. Cao, "Use of grating theories in integrated optics," J. Opt. Soc. Amer. A, vol. 18, pp. 2865-2875, 2001.

[7] J. P. Berenger, "A perfectly matched layer for the absorption of electromagnetic waves,” J. Comput. Phys., vol. 114, pp. 185-200, 1994.

[8] P. Bienstman and R. Baets, "Optical modeling of photonic crystals and VCSELs using eigenmode expansion and perfectly matched layers," Opt. Quantum Electron., vol. 33, pp. 327-341, 2001. 